\title{
Room Temperature Sol-Gel Fabrication and Functionalization for Sensor Applications
}

\author{
George HUYANG ${ }^{1,2}$, John CANNING ${ }^{1,2 *}$, Ingemar PETERMANN ${ }^{1,2}$, \\ David BISHOP $^{3}$, Andrew McDONAGH ${ }^{3}$, and Maxwell J. CROSSLEY ${ }^{1}$ \\ ${ }^{1}$ School of Chemistry, The University of Sydney, NSW 2006, Australia \\ ${ }^{2}$ Interdisciplinary Photonic Laboratories (iPL), School of Chemistry, 222 Madsen Building F09, The University of \\ Sydney, NSW 2006, Australia \\ ${ }^{3}$ University of Technology Sydney, Broadway NSW 2007, Australia \\ *Corresponding author: John CANNINGＥ-mail: john.canning@sydney.edu.au
}

\begin{abstract}
The structure and physical properties of a thin titania sol-gel layer prepared on silicon and silica surfaces were examined. Spectroscopic (FTIR, UV-VIS spectroscopy), refractive index (ellipsometry) and microscopic (light microscopy and SEM/EDS) tools were used to examine both chemical uniformity and physical uniformity of the sol-gel glass layers. The conditions for the fabrication of uniform layers were established, and room temperature dopant incorporation was examined. The absorption bands of porphyrin-containing titania sol-gel layers were characterized. By addition of a metal salt to the titania layer, it was possible to metallate the free-base porphyrin within and change the UV-VIS absorbance of the porphyrin, the basis of metal detection using porphyrins. The metalloporphyrins were detected by localized laser ablation inductive coupled mass spectroscopy (LA-ICP-MS), indicating fairly uniform distribution of metals across the titania surface.
\end{abstract}

Keywords: Sol-gel, titania, titanium dioxide, evanescent field, sensor, organic compound, spectroscopy, microscopy, surface functionalization, ablation inductive coupled mass spectroscopy

\section{Introduction}

Many of the chemical-based optical sensors currently being explored utilize a sensing molecule and its interaction with selected target molecules. This interaction facilitates a spectroscopic change that can be quantified using the Beer-Lambert law or variations of this principle. The sensitivity of the sensor can be enhanced by increasing the amount of interaction between the chemical and the electromagnetic fields associated with the optical sensor. Optical fiber based sensors are of increasing importance because of their potential for remote detection, free operation, distributed sensing and digital integration into smartgrid sensor networks [1]. Optical fibers have been well studied as the chemical sensor $[2,3]$. Of particular note is the use of porous structures that permit the active molecules to be adsorbed in close physical proximity to the exciting light, typically in an evanescent configuration [3] but direct field overlap is also feasible. These porous materials may be constructed from different kinds of sol-gels, including silica- and titania-based. Titania porous materials have a strong affinity for binding organic compounds, presumably in part a result of a greater quantity and larger range

Received: 23 June 2012 / Revised version: 5 July 2012

(C) The Author(s) 2012. This article is published with open access at Springerlink.com 
of oxide defect states. It absorbs very little visible light, has a higher refractive index than silica, and high porosity that allows for gases and liquids to enter the structure making it an excellent material for optical sensing in the waveguide or fiber form, for example.

We recently proposed a technique to coat the holes of a structured fiber with a titania sol-gel material, combining the benefits of its absorption properties with the fiber-based sensing technology [4] based on its affinity for binding $-\mathrm{OH}$ containing compounds [5]. Titania micron-thickness layers fabricated by sol-gel condensation have been extensively studied, with their spectroscopy and elemental composition characterized in detail, including within optical fibers. The high refractive index has been exploited to draw out the evanescent field to increase interaction with adsorbed materials, generating the resonator and whispering gallery mode enhancement whilst minimizing additional insertion and coupling loss to standard telecommunications fiber. Orders of magnitude improvement in sensitivity compared to conventional fiber sensors are obtained this way [6]. Generally, the construction of titania sol-gel components requires very specific conditions and control over preparation conditions $[7,8]$. We also recognized that for optical sensing it would be of immense practical interest to have a process for fabricating thin films at low temperatures so that a variety of materials suitable for sensing, in particular organic molecules, could be integrated directly into the glass. Therefore, we examined the feasibility of fabricating sol-gel layers without thermal processing or heat sintering of up to $900^{\circ} \mathrm{C}$, which is commonly applied to consolidate and stabilize standard sol-gel preparation [9]. In this work, we integrated and compared several organic systems, including porphyrins.

To characterize the sol-gel surface, the sol-gel deposition process was performed using surfaces such as silica glass slides and silicon wafers with oxide termination. By characterizing the properties of the titania layer, we evaluated their effectiveness for binding selective compounds when applied to other optical surfaces.

The porphyrins used for binding in this work have chemical design flexibility allowing for sensitive electronic and excitonic bandgap engineering. For sensing, the metallation of free-base porphyrins is an important area of research, as this process exhibits strong UV-VIS changes upon reaction. For example, the incorporation of a metal ion within its macrocycle enables a metal impurity sensor, increasingly important for water, environmental and industrial sensing. The integration of such metal sensing species with titania is therefore of great interest [10]. The uptake of metal ions $\left(\mathrm{Cu}^{2+}\right.$ and $\left.\mathrm{Zn}^{2+}\right)$ by a porphyrin-laced titania layer along with the distribution of both porphyrin and metal intake across the layer can be characterized using laser ablation inductive coupled plasma mass spectrometry (LA-ICP-MS). LA-ICP-MS uses a laser to ablate surfaces to generate aerosols, which are collected for elemental analysis by standard mass spectrometry. It has previously been used to detect metallated phthalocyanines, which have a similar chemical structure to porphyrins [11]. LA-ICP-MS has also been used to detect the presence of trace quantities of zinc and copper [12].

\section{Methodology}

The surfaces used were borosilicate glass slides and silicon oxide terminated silicon with an oxide thickness of $20 \mu \mathrm{m}$. Following conventional procedures, the slides and wafers were washed with $\mathrm{NH}_{3} / \mathrm{H}_{2} \mathrm{O}_{2} / \mathrm{H}_{2} \mathrm{O}$ (1:1:5) for surface activation, followed by washing with de-ionized water and drying under $\mathrm{N}_{2}$. The sol-gel layer was formed with $5 \%$ DuPont $^{\mathrm{TM}}$ TYZOR $^{\circledR}$ TPT (tetra-isopropyl titanate) in the isopropanol solution and left overnight for aging. The slides were spin-coated at $1000 \mathrm{rpm}$ for 60 seconds with the titania sol-gel, which resulted in a slide covered with a thin, faintly iridescent layer of titania. For organic treatment, samples were placed 
in a range of organic solutions for comparison (hydroxyporphyrin, rhodamine B, thymol blue and bromothymol blue) overnight. The slides and wafers were finally washed with isopropanol to remove unbound organic material and dried with $\mathrm{N}_{2}$. This cold-preparation method is a simplified version of the common procedure for preparing titania sol-gel where the annealing process is deliberately avoided.

Specific variations of the preparation conditions, designed to adjust the layer thickness, include a reduction in the concentration to $1 \%$, increase in the spin-rate to $6000 \mathrm{rpm}$, and heating the slide to $300{ }^{\circ} \mathrm{C}$. It should be noted that the $\mathrm{Si}$ surfaces (which have a thin $\mathrm{SiO}_{2}$ layer) were chosen as models for other surfaces containing $\mathrm{SiO}_{2}$ surfaces, e.g., optical fibers Samples were analyzed by Fourier transform infrared (FTIR) spectroscopy, UV-VIS, ellipsometry, light microscopy, scanning electron microscopy (SEM), and energy dispersive X-ray scattering (EDS).

The metallation process was performed differently for each sample using four distinct processes, two of which involved dual combination of reagents onto the sol gel layer (simulating a real sensing environment) and two of which involved direct deposition after the metal has been bound to the porphyrin. Table 1 and Scheme 1 summarize the metallation pathway and compounds used for metallation.

Table 1 Metallation for sol-gel/porphyrin samples.

\begin{tabular}{c|c|c}
\hline Sample & Compounds added & Metallation site \\
\hline 1 & Porphyrin $+\mathrm{Zn}(\mathrm{OAc})_{2} \cdot 2 \mathrm{H}_{2} \mathrm{O}$ solution & On sol-gel layer \\
2 & Porphyrin $+\mathrm{Cu}(\mathrm{OAc})_{2} \cdot \mathrm{H}_{2} \mathrm{O}$ solution & On sol-gel layer \\
3 & $\mathrm{Zinc}(\mathrm{II})$ porphyin & External \\
4 & Copper (II) porphyrin & External \\
\hline
\end{tabular}

\section{Results}

\subsection{Sol-gel layer characterization}

Light microscopy examination of the surface indicated a colorless layer at a micron-scale thickness formed by the centripetal force during spin coating causing the sol-gel layer to spread (Fig. 1) and assemble into a matrix of titanium oxide.

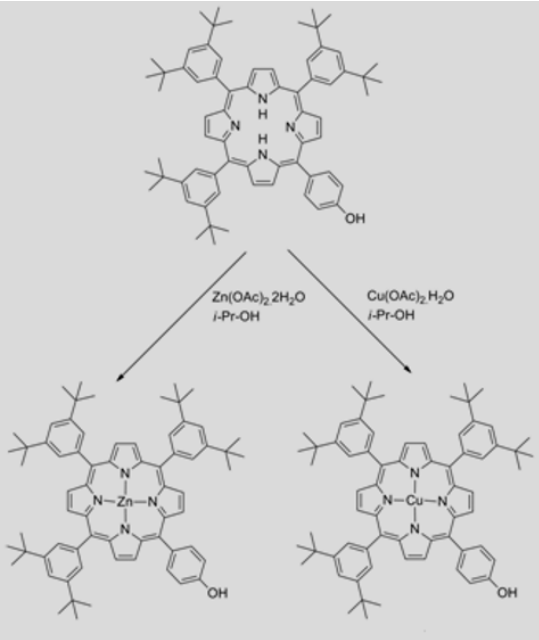

Scheme 1 Reaction pathway for the porphyrin metallation.

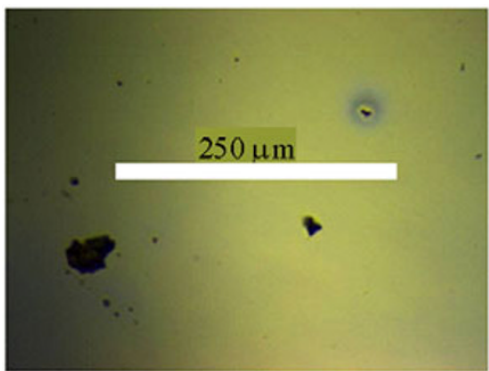

(a)
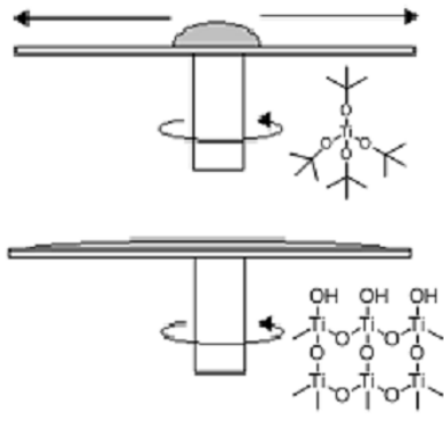

(b)

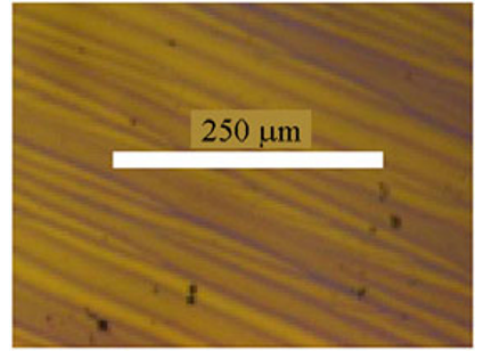

(c)

Fig. 1 Light microscope image of the titania sol-gel layer formed by spin-coating with the modeled spreading, comparing the center region (a) with particle aggregation over a smooth surface to the rim region, (b) illustrating the spincoating, and (c) showing aggregation into a grooved layer. 
However, a 2D view of the surface indicated several unwanted aggregations of titania particles and regions of groove-like features on the outer regions, possibly due to the liquid motion during spin-coating on the rectangular surface, leading to non-isotropic forces in different regions.

The titania layer was examined using FTIR spectroscopy, which has been used by Lopez et al. [13-15] to determine details of the sol-gel form structure, including the specific form of titania (based on different temperatures of annealing) and different $\mathrm{pH}$ conditions, including acids which would bind onto titania [13-15]. Different regions of the titania surface on silicon wafers and silicate microscope slides [Fig. 2(a)] were examined. Measurements on both surfaces showed the titania (Ti-OH) bending overtone at $1639 \mathrm{~cm}^{-1}$ [Fig. 2 (b)], comparable to the primary FTIR peak of titania powder [Fig. 2 (c)] in a solid matrix observed at $1637 \mathrm{~cm}^{-1}$. Other peaks included a broad O-H peak in the $3500 \mathrm{~cm}^{-1}-3000 \mathrm{~cm}^{-1}$ range and a Si-O band at $1000 \mathrm{~cm}$. A similar Ti-O peak in both surfaces confirmed that the films on silicate (borosilicate) slides and silicon (silicon oxide) wafers had similar chemical environments. The FTIR spectra varied little between different regions indicating a uniform titania coverage over the surface. This data confirmed that the process was reproducible on different silica-based surfaces.

\subsection{Refractive index measurement}

In our previous work using optical fibers, an important factor regarding the interaction of light with the titania structure was the evanescent field overlap between the fiber core and the titania sol-gel, which depended on the thickness and refractive index of the layer. The layer thickness and composition were measured by SEM (Fig. 3) of the sol-gel on a silicon wafer. The structure of these titania sol-gel layers appeared to be a thick, jelly-like covering of about $3 \mu \mathrm{m}$ over a thinner layer of titania of about 300-nm thickness. This was

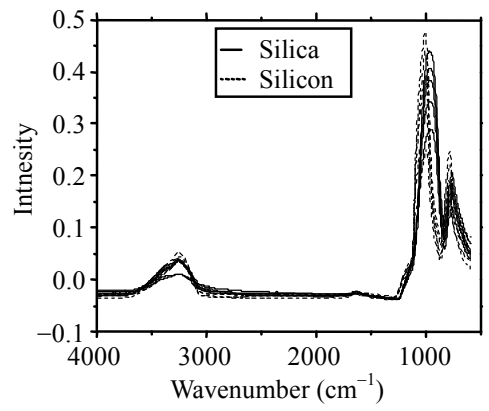

(a)

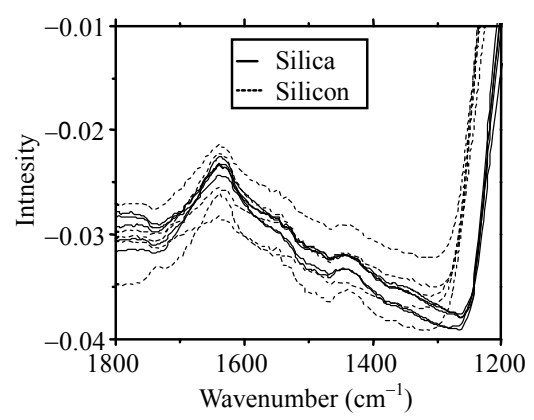

(b)

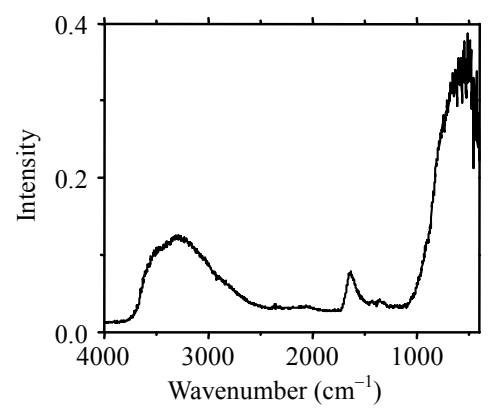

(c)

Fig. 2 FTIR spectra of (a) the titania sol-gel layer on borosilicate glass and thermal silicon oxide (weaker signal) at different regions of the microscope slide and silicon wafer surface, (b) the specific Ti-OH bending peak highlighted, and (c) a spectrum of a solid matrix of titanium oxide particles showing the same peaks.

confirmed by energy dispersive spectroscopy (EDS) that indicated the topmost layer had a much higher titanium and carbon content compared to the silicon and oxygen content below from the silicon wafer. With these thickness measurements, ellipsometry was used to determine the refractive index of the titania layer to a range of 1.9-2.1 over the VIS-NIR spectrum (Fig. 4). This value, whilst lower than the denser rutile (2.6), the most common form of titanium oxide, is much higher than that of silica 
despite the porosity of the sol-gel. This allows for light to be drawn from the silica into the titania film, and therefore also the evanescent field, when used with waveguides. It is these properties that allow for greater interactions between the bound chemicals and the light. This is due to the porous regions containing the air or organic material which can lower the overall refractive index of the layer.

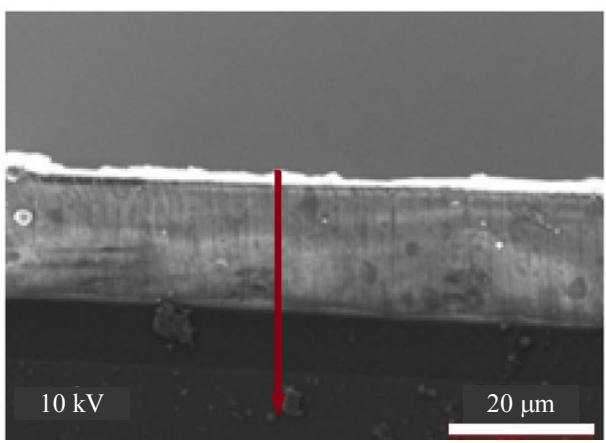

(a)
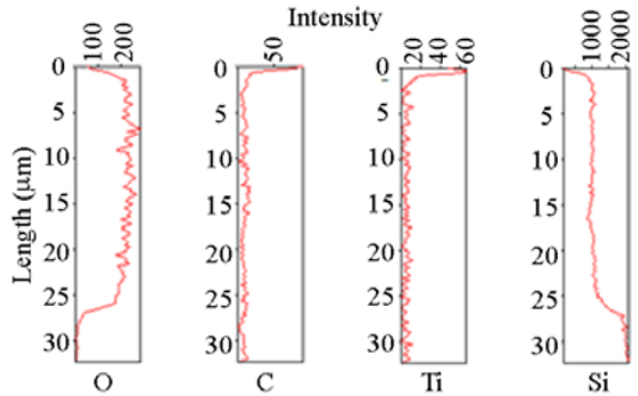

(b)

Fig. 3 SEM and EDS analysis of a sol-gel layer: (a) SEM cross-section of the titania sol-gel layer and (b) EDS line-scan profile from the top of the sol-gel layer to the middle of the silicon wafer (oxygen- $\mathrm{O}$, carbon- $\mathrm{C}$, titanium- $\mathrm{Ti}$, and silicon - Si are all detected).

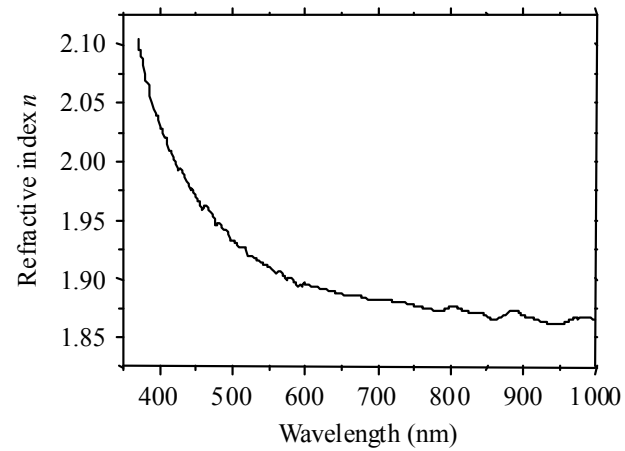

Fig. 4 Refractive index of the titania layer at the wavelength range of $370 \mathrm{~nm}-1000 \mathrm{~nm}$, indicating refractive index varying between 1.9 to 2.1 .

\subsection{Binding of organic compounds}

Titania-based sol-gels are well known to bind to a variety of organic compounds containing hydroxyl $(-\mathrm{OH})$ or carboxyl $(-\mathrm{COOH})$ groups. To consider the specific chemical binding, the binding between the

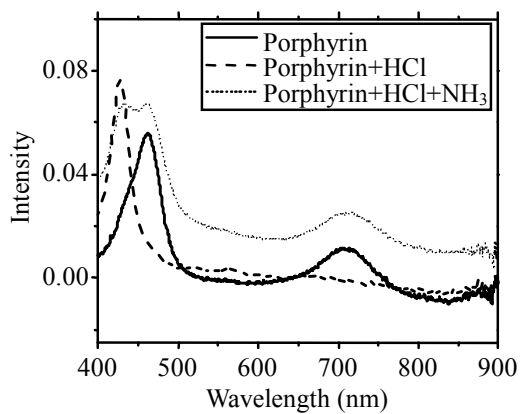

(a)

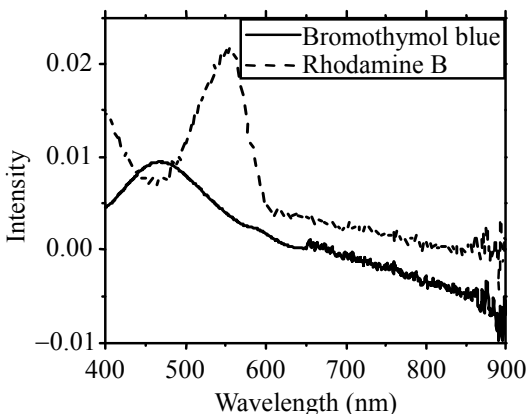

(b)

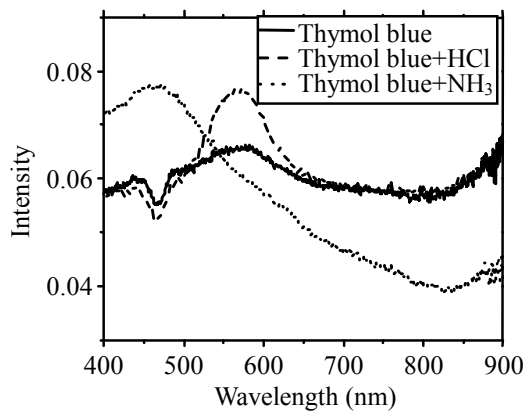

(c)

Fig. 5 Spectral measurements of various organic species under varying conditions as shown (a) UV-VIS spectra of the light passing through a titania sol-gel layer with hydroxyporphyrin, as well as its changes after acidifying and then basifying, (b) UV-VIS spectra of the light passing through a similar titania layer with rhodamine B and bromothymol blue, and (c) UV-VIS spectra of the light passing through a similar titania layer with thymol blue after acidifying and basifying. However, unlike the porphyrin, the basifying was done on a separate sample rather than immediately after acidification. 
titania surface and several organic systems hydroxyporphyrin, rhodamine $\mathrm{B}$, thymol blue, and bromothymol blue (chemical structures included in Fig. 5) - was investigated. These compounds were selected because of their relevant functional groups and high extinction coefficients, which could be changed by acidic/basic conditions (with the exception of rhodamine $\mathrm{B}$ which was selected because of its fluorescence).

Titania surfaces were immersed in solutions of these compounds and then analyzed by UV-VIS spectroscopy. The compounds have strong absorption bands in the UV-VIS range, but titania itself does not. The surfaces were washed to remove the unbound material, leaving only the compounds with strong chemisorption to the sol-gel. The hydroxyporphyrin, rhodamine $\mathrm{B}$ and thymol blue showed strong affinity for binding to the titania surface, given that their characteristic spectra were observed for all samples. The bromothymol blue showed a much weaker absorbance to the titania, indicating less absorbed material compared to the thymol blue. This is explained by examining its structure where the $-\mathrm{OH}$ group is sterically hindered by the bromine, indicating that bonding through $\mathrm{OH}$ occurs (see Scheme2). However, a small quantity of

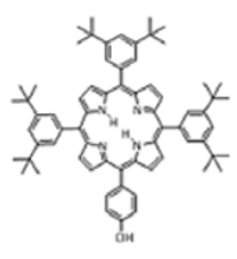

Hydrox yporphyrin

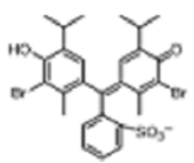

Bromothymol blue

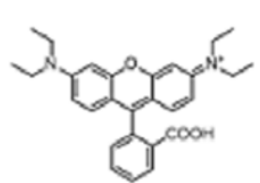

Rhoda mine $\mathrm{B}$

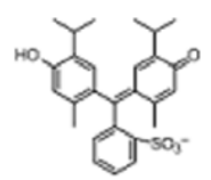

Thymol blue
Scheme 2 Chemical structure of the organic compounds used.

bromothymol blue was still bound directly to the titania, possibly due to a certain amount of physisorption between bromothymol blue and titania despite the lack of chemisorption. The substances bound onto the sol-gel show interaction with acidic hydrogen chloride $(\mathrm{HCl})$ and basic ammonia gas $\left(\mathrm{NH}_{3}\right)$. The lack of absorbance bands in the visible region allow for a complete spectroscopic analysis of only the compounds that are bound.

\subsection{Modifications to the sol-gel processing technique}

The sol-gel fabrication process used in this work lacked the standard heat treatment process, which is normally a requirement for the preparation of stable, higher quality sol-gel glassy films. The intention of making a highly porous layer was also a consideration, so that heat sintering of the titania, which would better solidify the sol-gel structure, was undesirable. However, after the process for sol-gel construction was optimized, the possibility of reducing the layer thickness was then considered.

SEM images [Fig. 6 (a)] showed that much of the deposited sol-gel was a gel-like layer over a more structured layer. Although the thicker gel-like layer had the desired porosity, some consideration was also given on how to reduce the layer thickness. The sol-gel preparation was modified to have lower titania concentration, which led to a less-uniform coverage of the surface [Fig. 6(b)]. Heat treatment to $300{ }^{\circ} \mathrm{C}$ [Fig. 6(c)] appeared to change the layer, making its structure more solid rather than gel-like. Another method for controlling the layer thickness was to increase the flow rate of the sol-gel. This was previously done in a fiber by using a pressure chamber, but on the slide surface this could be done by increasing the spin-coating speed. The relative centrifugal force $F$ of the sol-gel should be proportional to the rotor radius $R$ and the square of the spin rate $S$. Therefore, a six-fold increase in the spin-rate would lead to an equivalent of a thirty-six times greater force. This resulted in a much thinner layer [Fig. 6(d)]. The principle of using a greater force, and by extension, a greater flow speed, indicates that layer thickness can be controlled using this process. However, for the optical fiber applications, much like the limitation of heating the fiber, to get this quantity of force on the fiber 
cross-section would require several thousand atmospheres of pressure and so may be unfeasible.

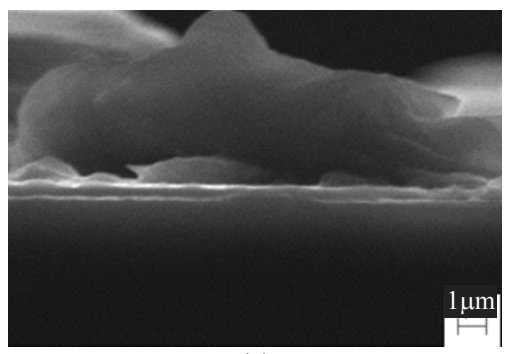

(a)

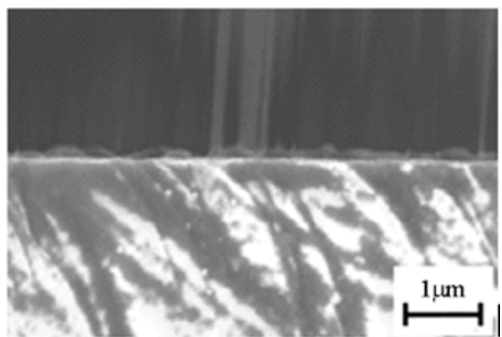

(b)

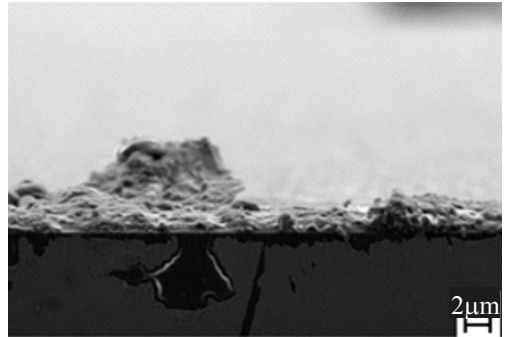

(c)

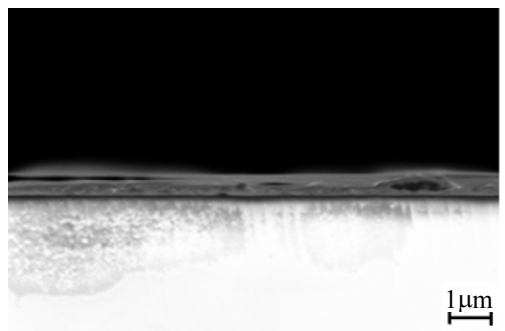

(d)

Fig. 6 SEM images of sol-gel layers: (a) the SEM image of the titania sol-gel layer with 5\% TPT concentration (standard preparation conditions), (b) SEM scan of the titania sol-gel with $1 \%$ TPT concentration, (c) SEM scan of the titania with heating to $300{ }^{\circ} \mathrm{C}$, and (d) SEM scan of the titania sol-gel layer with spin-coating at $6000 \mathrm{RPM}$.

\subsection{Analysis by LA-ICP-MS}

The UV-VIS spectra of samples 1-4 are shown in Fig. 7. A shift in the Soret band observed with samples (1) and (2) indicated that metallation had occurred by absorbing metal into the sol-gel, where it reacted with the porphyrin within. Samples (3) and (4), which contained the externally metallated metalloporphyrins, were compared with samples (1) and (2) and exhibited the same shift in the Soret band. Thus, in both reaction routes the metallated porphyrins were attached to the titania. The UV-VIS spectra confirmed that the pathway of samples (1) and (3) formed the same final product, and likewise with samples (2) and (4).

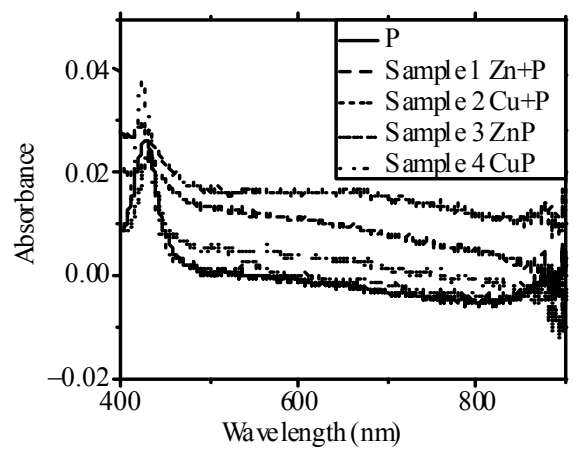

Fig. 7 UV-VIS transmission spectra of the titania sol-gel slide with the porphyrin starting material $(\mathrm{P})$ and its equivalent metallatoporphyrins from samples 1-4.

LA-ICP-MS measurements were carried out by taking multiple line-scans on the titania layer, with $2 \mathrm{~mm}$ between each scan. The elements that were measured included titanium (Fig. 8), zinc, and copper (Fig. 9). Multiple line-scans for each sample were compared to map the elemental composition of the surface. The data showed that the titania composition was relatively constant throughout the slide for samples (1) and (2) but linearly decreased in quantity from one end to the other in samples (3) and (4). The results indicated a similar quantity of titania overall, although a gradual decrease in samples (3) and (4) was observed, suggesting uniform layer formation with no obvious aggregation.

The metal ions had considerable fluctuation in concentration in samples (1) and (2) in contrast to samples (3) and (4). This was evidence that aggregation had occurred unevenly using the method of preparation of samples (1) and (2). The presence of metal ions in samples (3) and (4) confirmed successful metallation of the porphyrin, as the sol-gel layer was not previously in direct 

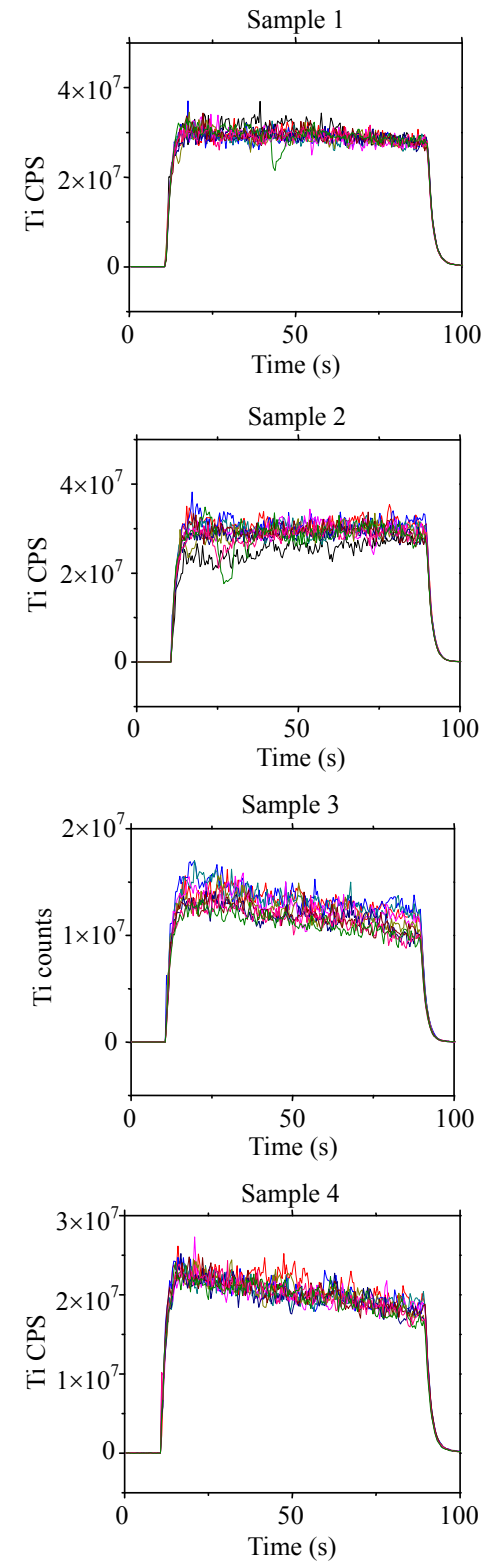

Fig. 8 LA-ICP-MS of samples $1-4$, indicating the Ti quantity for multiple lines scans across each sample.

contact with the metal ion. The only source of metals was from the metalloporphyrins. The metal quantity detected by LA-ICP-MS was also compared and is shown in Fig. 10. Samples (1) and (2), which had the porphyrin added first and then metal salt, were closer to a realistic sensor as it indicated interaction between porphyrin and metal ion. On these samples, there was a much larger quantity of metals detected on the surface. This might be attributed to direct absorption of metal salts onto the titania, indicating that the activity of the titania itself might be higher than that of the porphyrin. Given the highly porous structure of the sol-gels, which imparted a large surface area, this was quite likely. The amount of metal ions in solution greatly exceeded the amount of porphyrin, which required only one metal per molecule. This indicated that porphyrin intake was generally not high and that the metal affinity was non-uniform across the titania; which might in turn indicated that the porosity was non-uniform although the titania layer itself might be.
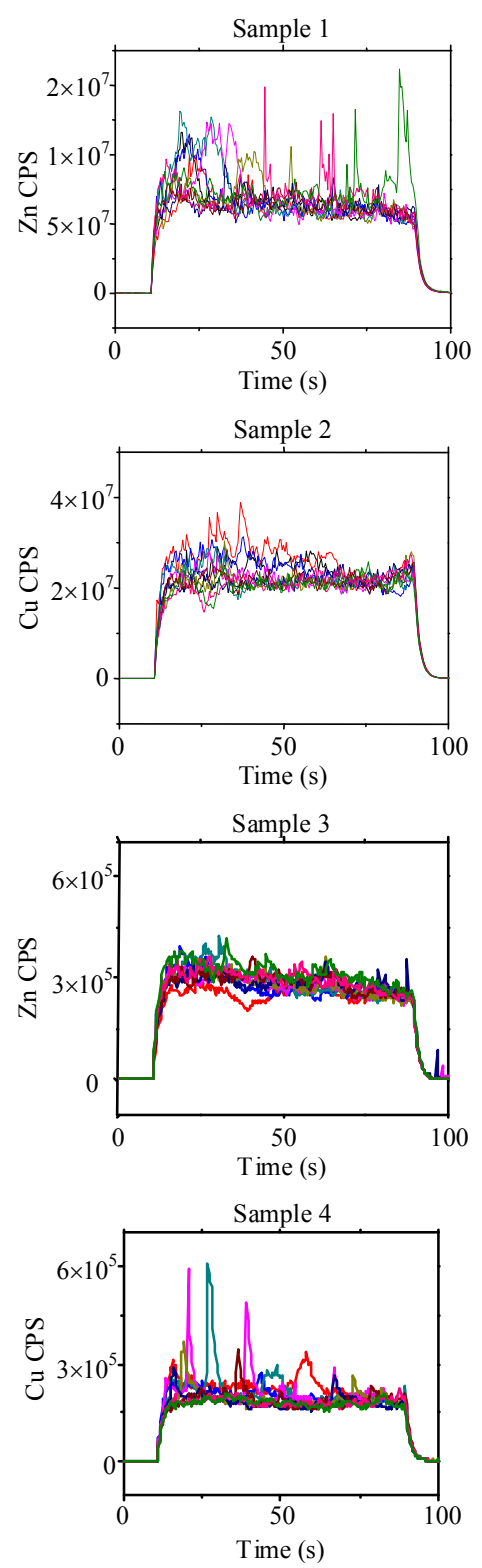

Fig. 9 LA-ICP-MS of samples 1-4, indicating the $\mathrm{Zn}$ and $\mathrm{Cu}$ quantity and distribution for multiple lines scans across each sample. 


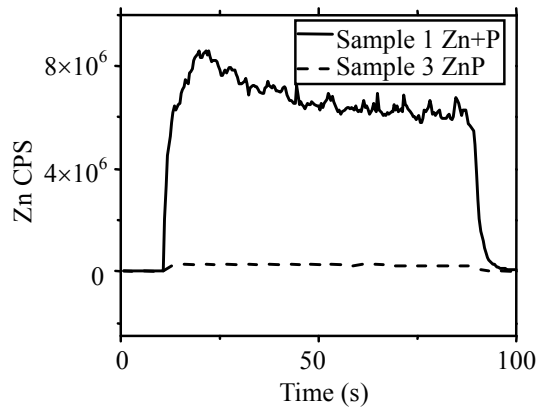

(a)

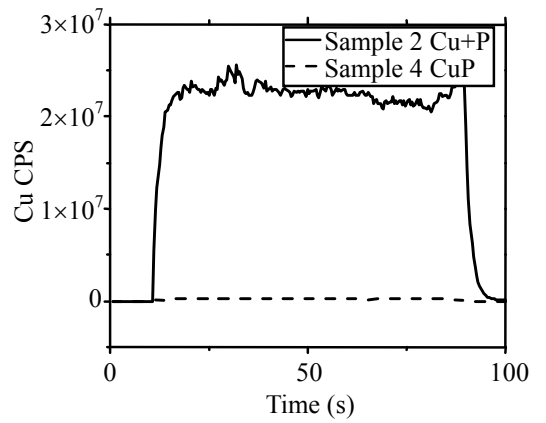

(b)

Fig. 10 LA-ICP-MS results comparing the quantity of (a) $\mathrm{Zn}$ on samples 1-3 and (b) $\mathrm{Cu}$ on samples 2-4.

\section{Conclusions}

In conclusion, we have demonstrated the construction of titania-based sol-gel layers on silicate and silicon oxide surfaces using a room temperature preparation process that is simpler than conventional sol-gel construction techniques and more suited for use with our optical components. The optical properties of sol-gel layers, such as transmission spectra and the binding selectivity, were measured. The structure of these sol-gel layers could also be ideally controlled, both with physical modifications to change the layer thickness, and also chemically by functionalizing reactions on the desired organic compounds for binding to the titania. Free-base hydroxyphenyl porphyrins bound onto titania sol-gel layers were successfully metallated within the sol-gel layers, confirmed by a shift in the Soret band. Elemental analysis of the surfaces by LA-ICP-MS confirmed that the distribution of metal over the surface was reasonably uniform when directly metalled porphyrins were used. In two samples, the competing interactions between porphyrins and metals when reagents were mixed directly on the surface led to significant intake of metal salts into the titania matrix itself, where it was strongly bound. Upon metal uptake, the porphyrin was subsequently metallated within the sol-gel layer and showed a shift in the Soret band, demonstrating the basis of a simple metal sensor.

The aggregation of the metal observed in samples (1) and (2) indicated non-uniform affinity or porosity along the titania layer, in spite of its own uniformity. Although the measurements were restricted to accessible films on slides, they provided useful insight into the likelihood of metal uptake. Of particular importance were the challenges of separating out and distinguishing porphyrin and titania-based attachments either in the film, waveguide or structured optical fiber, for example. samples (3) and (4) indicated that external metallation was necessary if direct metal-titania interactions were avoided (in some cases these might themselves be more useful). This was very much dependent on the type of the chemical sensor being explored. Optimization of these sol-gel layers for specific optical devices, such as optical fibers, might involve some additional mechanical manipulation that may not be possible on surfaces other than slides. Nevertheless, the work here suggests that it is possible to fabricate room temperature sol gel layers of sufficient robustness to warrant further exploration and application.

\section{Acknowledgment}

Australian Research Council Grant FT110100116 supported this work. Agilent Technologies are acknowledged for their support.

Open Access This article is distributed under the terms of the Creative Commons Attribution License which permits any use, distribution, and reproduction in any medium, provided the original author(s) and source are credited. 


\section{References}

[1] J. Canning, "Properties of specialist fibers and Bragg gratings for optical fiber sensors," Journal of Sensors, vol. 2009, pp. 871580, 2009.

[2] R. Gupta and N. K. Chaudhury, "Entrapment of biomolecules in sol-gel matrix for applications in biosensors: problems and future prospects," Biosensors Bioelectronics, vol. 22, no. 11, pp. 2387-2399, 2007.

[3] P. C. A. Jerónimoa, A. N. Araújo, and M. C. B. S. M. Montenegro, "Optical sensors and biosensors based on sol-gel films", Talanta, vol. 72, no. 1, pp. 13-27, 2007.

[4] G. Huyang, J. Canning, M. L. Åslund, D. Stocks, T. Khoury, and M. J. Crossley, "Evaluation of optical fiber microcell reactor for use in remote acid sensing," Optics Letters, vol. 35, no. 6, pp. 817-819, 2010.

[5] J. Y. Wen and G. L. Wilkes, "Organic/Inorganic hybrid network materials by the sol-gel approach," Chemistry of Materials, vol. 8, no. 8, pp. 1667-1681, 1996.

[6] J. Canning, W. Padden, D. Boskovic, M. Naqshbandi, H. de Bruyn, and M. J. Crossley, "Manipulating and controlling the evanescent field within optical waveguides using high index nanolayers," Optical Materials Express, vol. 1, no. 2, pp. 192-200, 2011.

[7] S. J. Park, Y. C. Kang, J. Y. Park, E. A. Evans, R. D. Ramsier, and G. G. Chase, "Physical characteristics of titania nanofibers synthesized by sol-gel and electrospinning techniques," Journal of Engineered Fibers and Fabrics, vol. 5, no. 1, pp. 50-56, 2010.

[8] Z. Miao, D. Xu, J. Ouyang, G. Guo, X. Zhao, and
Y. Tang, "Electrochemically induced sol-gel preparation of single-crystalline $\mathrm{TiO}_{2}$ nanowires," Nano Letters, vol. 2, no. 7, pp. 717-720, 2002.

[9] C. C. Wang and J. Y. Ying, "Sol-gel synthesis and hydrothermal processing of anatase and rutile titania nanocrystals," Chemistry of Materials, vol. 11, no. 11, pp. 3113-3120, 1999.

[10] D. Delmarre, R. Méallet, C. Bied-Charreton, and R. B. Pansu, "Heavy metal ions detection in solution, in sol-gel and with grafted porphyrin monolayers," Journal of Photochemistry Photobiology A: Chemistry, vol. 124, no. 1-2, pp. 23-28, 1999.

[11] T. Rawling, C. Austin, D. Hare, P. A. Doble, H. M. Zareie, and A. M. McDonagh, "Thin films of ruthenium phthalocyanine complexes," Nano Research, vol. 2, no. 9, pp. 678-687, 2009.

[12] C. Austin, D. Hare, T. Rawling, A. M. McDonagh, and P. J. Doble, "Quantification method for elemental bio-imaging by LA-ICP-MS using metal spiked PMMA films," Journal of Analytical Atomic Spectrometry, vol. 25, no. 5, pp. 722-725, 2010.

[13] T. Lopez, E. Sanchez, P. Bosch, Y. Meas, and R. Gomez, "FTIR and UV-Vis (diffuse reflectance) spectroscopic characterization of $\mathrm{TiO}_{2}$ sol-gel," Materials Chemistry and Physics, vol. 32, no. 2, pp. 141-152, 1992.

[14] T. Lopez, E. Ortiz, R. Gomez, and M. Picquart, "Amorphous sol-gel titania modified with heteropolyacids," Journal of Sol-Gel Science and Technology, vol. 37, no. 3, pp. 189-193, 2006.

[15] T. Lopez, E. Ortiz-Ilas, E. Vinogradova, J. Manjarrez, J. A. Azamar, J. J. Alvarado-Gil, et al., "Structural, optical and vibrational properties of sol-gel titania/valproic acid reservoirs," Optical Materials, vol. 29, no. 1, pp. 82-87, 2006. 